\title{
Critical behaviors of sheared frictionless granular materials near jamming transition
}

\author{
Michio Otsuki ${ }^{1}$ and Hisao Hayakawa ${ }^{21}$ \\ 11 Department of Physics and Mathematics, Aoyama Gakuin University, \\ 5-10-1 Fuchinobe, Sagamihara, Kanagawa, 229-8558, Japan. \\ 2 Yukawa Institute for Theoretical Physics, Kyoto University, \\ Kitashirakawaoiwake-cho, Sakyo-ku, Kyoto 606-8502, Japan.
}

\begin{abstract}
Critical behaviors of sheared dense and frictionless granular materials in the vicinity of the jamming transition are numerically investigated. From the extensive molecular dynamics simulation, we verify the validity of the scaling theory near the jamming transition proposed by Otsuki and Hayakawa (Prog. Theor. Phys., 121, 647 (2009)). We also clarify the critical behaviors of the shear viscosity and the pair correlation function based on both a mean field theory and the simulation.

PACS numbers: 45.70.-n, 05.70.Jk, 47.50.-d
\end{abstract}

\section{INTRODUCTION}

Let us consider mechanical properties of grains which are packed in a container. When the density is low enough and the effect of gravity is negligible, there is no pressure acting on the wall of the container. However, when the density exceeds a critical value, the pressure acting on the wall becomes finite. Such kind of transition for the rigidity or the stress is known as the jamming transition.

Jamming is a key concept to characterize the transition of athermal systems such as granular materials [1], colloidal suspensions [2], emulsions and forms [3]. Liu and Nagel suggested that a unifying description might be possible to cover both glass transition in thermal systems and athermal jamming transition [4]. Indeed, there are many similarities between the jamming and the glass transition. For example, the rheological properties of the jammed systems [5, 6] are similar to those of glassy materials [7], and the granular materials near the jamming transition point (point J) exhibit the dynamical heterogeneity which is one of the characteristics of glassy materials $[6,[8,[9,10,11,12,13]$. From these similarities, the mode coupling theory for glassy materials [14, 15, 16] is applied to granular materials, but it fails to describe the jamming transition [17].

Recent studies have revealed that the jamming is the transition to appear in the pressure, the coordination number, the elastic moduli [18, 19], and the soft modes [20]. In particular, Olsson and Teitel [6] , and Hatano [21] numerically found scaling relations for the shear stress, the pressure, and the kinetic temperature, which are characterized by some critical exponents. A similar scaling relation is observed in the simulation of Josephson junction arrays 22]. These scaling relations indicate that jamming is a continuous transition in which the stress is zero at the critical point. In order to understand the critical behaviors of the jamming transition, it is important to determine the critical exponents. In the previous paper [23], the authors theoretically obtained the critical exponents for the scaling relations of the shear stress, the kinetic temperature and the characteristic frequency, which characterizes the collisional energy loss, for sheared frictionless granular materials. They also numerically verified the validity of their theoretical predictions for the linear spring model [23].

In this paper, we will verify the validity of the predictions in Ref. [23] for sheared frictionless granular materials in various situations. We will also discuss the behaviors of the viscosity and the pair correlation function in details. In the next section, we will summarize the theoretical predictions by the present authors [23]. In Sec. III, we will compare the results of our extensive simulations with the theoretical predictions. Section III consists of five subsections: Section IIIA will be devoted to the explanation of the setup of our simulations, in Sec IIB we will present various scaling plots to verify the scaling theory, in Sec. IIIC we will discuss the force law dependence of the critical exponents, we will explain the density dependence of critical variables in Sec. IIID We will explain the results for nearly elastic cases in Sec. IIIE Section IV will be devoted to the explanation of critical properties of the pair correlation function in the vicinity of the jamming transition, which was not discussed in Ref. 23]. In Sec. V] we will discuss and conclude our results. In Appendix A, we will summarize the method for the theoretical prediction which contains some generalized results beyond Ref. [23] based on a different method for the derivation. In Appendix B, we will derive some relations, which are necessary to discuss the critical behavior of the pair correlation function.

\section{MEAN FIELD THEORY}

In this section, we briefly summarize the theoretical results in Ref. [23]. Let us consider $D$-dimensional granular assemblies under an uniform shear with shear rate $\dot{\gamma}$. The system includes $N$ grains, each of which has the identical mass $m$. The packing fraction of the system and the critical fraction at point $\mathrm{J}$ are respectively denoted by $\phi$ and $\phi_{J}$. Throughout this paper, we assume that granular particles are frictionless, where any contact force acts along the line to connect two centers of mass of 
contacting grains. In most of our arguments, we assume that the elastic interaction between the grain $i$ located at $\boldsymbol{r}_{i}$ and the grain $j$ at $\boldsymbol{r}_{j}$ is given by

$$
f_{\mathrm{el}}\left(r_{i j}\right)=k \Theta\left(\sigma_{i j}-r_{i j}\right)\left(\sigma_{i j}-r_{i j}\right)^{\Delta},
$$

where $k$ and $r_{i j}$ are the elastic constant and the distance between the grains $r_{i j} \equiv\left|\boldsymbol{r}_{i j}\right|=\left|\boldsymbol{r}_{i}-\boldsymbol{r}_{j}\right|$, respectively. $\sigma_{i j}=\left(\sigma_{i}+\sigma_{j}\right) / 2$ is the average of the diameter of the grain $i$ and the grain $j$ with diameters $\sigma_{i}$ and $\sigma_{j} . \Theta(x)$ is the Heaviside step function satisfying $\Theta(x)=1$ for $x \geq 0$ and $\Theta(x)=0$ for otherwise. Many researchers use the linear spring model $(\Delta=1)$ associated with the viscous contact force

$$
f_{\mathrm{vis}}\left(r_{i j}, v_{i j, \mathrm{n}}\right)=-\eta \Theta\left(\sigma_{i j}-r_{i j}\right) v_{i j, \mathrm{n}},
$$

where $\eta$ is the viscous parameter. Here, $v_{i j, \mathrm{n}}$ is the relative normal velocities between the grains given by $v_{i j, \mathrm{n}} \equiv\left(\boldsymbol{v}_{i}-\boldsymbol{v}_{j}\right) \cdot \boldsymbol{r}_{i j} / r_{i j}$, where $\boldsymbol{v}_{i}$ and $\boldsymbol{v}_{j}$ are the velocities of the grain $i$ and the grain $j$, respectively. On the other hand, the Herzian model (Eq.(1) with $\Delta=3 / 2$ ) associated with the corresponding viscous contact force

$$
f_{\mathrm{vis}}\left(r_{i j}, v_{i j, \|}\right)=-\eta \Theta\left(\sigma_{i j}-r_{i j}\right) \sqrt{\sigma_{i j}-r_{i j}} v_{i j, \mathrm{n}}
$$

is more realistic one for three-dimensional spheres.

In the previous paper [23], we introduced the scaling relations

$$
\begin{aligned}
& T=A_{T, D}|\Phi|^{x_{\Phi}} \mathcal{T}_{ \pm}\left(t_{D} \frac{\dot{\gamma}}{|\Phi|^{x_{\Phi} / x_{\gamma}}}\right) \\
& S=A_{S, D}|\Phi|^{y_{\Phi}} \mathcal{S}_{ \pm}\left(s_{D} \frac{\dot{\gamma}}{|\Phi|^{y_{\Phi} / y_{\gamma}}}\right) \\
& P=A_{P, D}|\Phi|^{y_{\Phi}^{\prime}} \mathcal{P}_{ \pm}\left(p_{D} \frac{\dot{\gamma}}{|\Phi|^{y_{\Phi}^{\prime} / y_{\gamma}^{\prime}}}\right) \\
& \omega=A_{W, D}|\Phi|^{y_{\Phi}} \mathcal{W}_{ \pm}\left(w_{D} \frac{\dot{\gamma}}{|\Phi|^{y_{\Phi} / y_{\gamma}}}\right)
\end{aligned}
$$

where $\Phi \equiv \phi-\phi_{J}$. The kinetic temperature $T$, the shear stress $S$, and the pressure $P$ are respectively given by [24]

$$
\begin{aligned}
T= & \frac{1}{N D}\left\langle\sum_{i=1}^{N} \frac{\left|\boldsymbol{p}_{i}\right|^{2}}{2 m}\right\rangle \\
S= & -\frac{1}{V}\left\langle\sum_{i}^{N} \sum_{j>i} \frac{r_{i j, x} r_{i j, y}}{r_{i j}}\left[f_{\mathrm{el}}\left(r_{i j}\right)+f_{\mathrm{vis}}\left(r_{i j}, v_{i j, \|}\right)\right]\right\rangle \\
& -\frac{1}{V}\left\langle\sum_{i=1}^{N} \frac{p_{x, i} p_{y, i}}{2 m}\right\rangle \\
P= & \frac{1}{D V}\left\langle\sum_{i}^{N} \sum_{j>i} r_{i j}\left[f_{\mathrm{el}}\left(r_{i j}\right)+f_{\mathrm{vis}}\left(r_{i j}, v_{i j, \|}\right)\right]\right\rangle \\
& +\frac{1}{D V}\left\langle\sum_{i=1}^{N} \frac{\left|\boldsymbol{p}_{i}\right|^{2}}{2 m}\right\rangle
\end{aligned}
$$

where we have introduced $\boldsymbol{p}_{i} \equiv m\left[\boldsymbol{v}_{i}-\boldsymbol{c}\left(\boldsymbol{r}_{i}\right)\right]$ with the average velocity $c_{\alpha}(\boldsymbol{r})=\dot{\gamma} y \delta_{\alpha, x}$ at the position $\boldsymbol{r}, V$ is the volume of the system, and $\langle\cdot\rangle$ represents the ensemble average. The characteristic frequency $\omega$ is defined by

$$
\omega \equiv \frac{\dot{\gamma} S}{n T}
$$

where $n$ is the number density. This $\omega$ is reduced to the collision frequency in the unjammed phase determined by the balance between the viscous heating and the collisional energy loss. $\mathcal{T}_{+}(x), \mathcal{S}_{+}(x), \mathcal{P}_{+}(x)$ and $\mathcal{W}_{+}(x)$ are the scaling functions above point $\mathrm{J}$ (in the jammed phase). On the other hand, the scaling functions below point $\mathrm{J}$ (in the unjammed phase) are given by $\mathcal{T}_{-}(x)$, $\mathcal{S}_{-}(x), \mathcal{P}_{-}(x)$ and $\mathcal{W}_{-}(x)$. Equations (41)-(7) contain the critical exponents $x_{\phi}, x_{\gamma}, y_{\phi}, y_{\gamma}, y_{\phi}^{\prime}, y_{\gamma}^{\prime}, z_{\phi}$, and $z_{\gamma}$. It should be noted that $A_{T, D}, A_{S, D}, A_{W, D}, A_{P, D}, t_{D}$, $s_{D}, w_{D}$, and $p_{D}$ are the constants depending only on the dimension $D$.

From the scaling theory explained in Appendix A, there are the scaling relations in the unjammed phase in the limit $\dot{\gamma} \rightarrow 0$ as

$$
\begin{array}{ll}
T \sim \dot{\gamma}^{2}|\Phi|^{x_{\phi}\left(1-2 / x_{\gamma}\right)}, \quad S \sim \dot{\gamma}^{2}|\Phi|^{y_{\phi}\left(1-2 / y_{\gamma}\right)} \\
P \sim \dot{\gamma}^{2}|\Phi|^{y_{\phi}^{\prime}\left(1-2 / y_{\gamma}^{\prime}\right)}, \quad \omega \sim \dot{\gamma}|\Phi|^{z_{\phi}\left(1-1 / z_{\gamma}\right)} .
\end{array}
$$

Similarly, $T, S, P$, and $\omega$ satisfy

$$
\begin{aligned}
& T \sim \dot{\gamma}|\Phi|^{x_{\phi}\left(1-1 / x_{\gamma}\right)}, \quad S \sim|\Phi|^{y_{\phi}}, \\
& P \sim|\Phi|^{y_{\phi}^{\prime}}, \quad \omega \sim|\Phi|^{z_{\phi}},
\end{aligned}
$$

in the zero shear limit of the jammed phase. The scaling relations at point J, i.e. $\Phi \simeq 0$, are given by

$$
T \sim \dot{\gamma}^{x_{\gamma}}, \quad S \sim \dot{\gamma}^{y_{\gamma}}, \quad P \sim \dot{\gamma}^{y_{\gamma}^{\prime}}, \quad \omega \sim \dot{\gamma}^{z_{\gamma}} .
$$

As explained in Appendix A and Ref. [23], the critical exponents are given by

$$
\begin{aligned}
& x_{\Phi}=2+\Delta, \quad x_{\gamma}=\frac{2 \Delta+4}{\Delta+4}, \quad y_{\Phi}=\Delta, \quad y_{\gamma}=\frac{2 \Delta}{\Delta+4}, \\
& y_{\Phi}^{\prime}=\Delta, \quad y_{\gamma}^{\prime}=\frac{2 \Delta}{\Delta+4}, \quad z_{\Phi}=\frac{\Delta}{2}, \quad z_{\gamma}=\frac{\Delta}{\Delta+4} .
\end{aligned}
$$

Note that the scaling of the pressure and the determination of the exponents $y_{\gamma}^{\prime}$ were not discussed in Ref. [23]. The derivations which are a little different from the original one are explained in Appendix A. We should stress that the exponents are independent of the spatial dimension $D$, and the characteristic feature of the jamming transition is the $\Delta$-dependence of the critical exponents. Indeed, O'Hern et al. found that the pressure behaves as $P \sim|\Phi|^{\Delta}$ for unsheared jammed systems in the vicinity of the jamming point. Hatano also indicated that the critical exponents for the sheared granular materials differ in the cases of $\Delta=1$ and $3 / 2$. Explicit $\Delta$-dependence of the exponents in Eq. (15) except for $y_{\gamma}^{\prime}$ for the sheared granular materials was obtained in Ref. [23]. It should be noted that our theory can be generalized to the case of the contact force

$$
f_{\mathrm{el}}\left(r_{i j}\right)=\Theta\left(\sigma_{i j}-r_{i j}\right) \mathcal{F}\left(r_{i j}\right)
$$


Here, the exponent $\Delta$ in Eq. (15) should be determined from the relation $\lim _{r_{i j} \rightarrow \sigma_{i j}} \mathcal{F}\left(r_{i j}\right) \propto\left(\sigma_{i j}-r_{i j}\right)^{\Delta}$ for this case. If we use an analytic function $\mathcal{F}\left(r_{i j}\right)$ such as the repulsive Lennard-Jones force

$$
\mathcal{F}\left(r_{i j}\right)=\frac{12 \epsilon}{\sigma_{i j}}\left[\left(\frac{\sigma_{i j}}{r_{i j}}\right)^{13}-\left(\frac{\sigma_{i j}}{r_{i j}}\right)^{7}\right],
$$

the value of $\Delta$ should be $\Delta=1$, because we can use the expansion $\mathcal{F}\left(r_{i j}\right) \simeq \mathcal{F}^{\prime}\left(\sigma_{i j}\right)\left(\sigma_{i j}-r_{i j}\right)+\mathcal{F}^{\prime \prime}\left(\sigma_{i j}\right) / 2\left(\sigma_{i j}-\right.$ $\left.r_{i j}\right)^{2}+\cdots$. Note that Eq. (17) differs from the usual form for the Lennard-Jones potential in order to satisfy $\mathcal{F}\left(\sigma_{i j}\right)=0$.

\section{COMPARISON BETWEEN THEORY AND SIMULATION}

In this section, let us verify the validity of the theory 23] by the molecular dynamics simulation. As stated in Introduction, we have already confirmed that all of our results of the simulation for $\Delta=1$ under one size distribution of grains with fixing the strength of inelasticity are consistent with the theory [23], but nobody has checked its validity for general $\Delta$ under various situations. Thus, we will perform extensive simulations with changing $\Delta$, the size distribution, and the inelasticity etc.

In the first part (IIIA), we explain the details of our model. In the second part (IIIB), we demonstrate the validity of the scaling theory given by Eqs. (4)-(17) with Eq. (15) from the test of scaling plots under various conditions. In the third part (IIIC), we check the theory for the $\Delta$-dependence of the critical exponents. In the fourth part (IIID), we discuss $\Phi$-dependences of the characteristic frequency $\omega$ in the jammed region and the viscosity $\mu=S / \dot{\gamma}$ in the unjammed region. In the last part (IIIE), we explain the behaviors for nearly elastic cases, while the results in the first five parts are obtained for strongly dissipative cases.

\section{A. Setup}

We examine three different systems on dispersion of diameters of grains. The first system we call the polydisperse system consists of four types of grains, and the diameters of grains are $0.7 \sigma_{0}, 0.8 \sigma_{0}, 0.9 \sigma_{0}$ and $\sigma_{0}$, where the number of each type of grains is $N / 4$. The polydisperse system has been studied in Ref. [23], where the critical fraction is estimated as $\phi_{J}=0.84285$ for $D=2$, $\phi_{J}=0.64455$ for $D=3$ or $\phi_{J}=0.4615$ for $D=4$ [23]. The second system which we call the bidisperse system consists of two types of grains. The diameters of grains are $5 \sigma_{0} / 7$ and $\sigma_{0}$, where the number of each type of grains is $N / 2$. The bidisperse system has been studied by many researchers [18, 19, 25, 26], and the critical density $\phi_{J}$ is known as 0.648 for $D=3$ by the numerical simulation of static granular packings [18]. The final system we call the monodisperse system consists of only one type of particles, whose diameters are $\sigma_{0}$. The critical density $\phi_{J}$ of the monodisperse system is believed to be 0.639 for $D=3$ from a numerical simulation [19].

The time evolution equations of the position $\boldsymbol{r}_{i}$ and the velocity $\boldsymbol{v}_{i}$ of the $i$ th particle are given by

$$
\begin{aligned}
\frac{d \boldsymbol{r}_{i}}{d t} & =\boldsymbol{v}_{i}, \\
m \frac{d \boldsymbol{v}_{i}}{d t} & =\sum_{j \neq i}\left\{f_{\mathrm{el}}\left(r_{i j}\right)+f_{\mathrm{vis}}\left(r_{i j}, v_{i j, \mathrm{n}}\right)\right\} \frac{\boldsymbol{r}_{i j}}{r_{i j}},
\end{aligned}
$$

where the elastic force $f_{\mathrm{el}}\left(r_{i j}\right)$ is given by Eq. (1) for most of cases except for the case of the repulsive LennardJones potential. $f_{\mathrm{vis}}\left(r_{i j}, v_{i j, \mathrm{n}}\right)$ given by Eqs. (2) or (3) is the viscous contact force between particles. In order to realize an uniform velocity gradient $\dot{\gamma}$ in $y$ direction and macroscopic velocity only in the $x$ direction, we adopt the Lees-Edwards boundary conditions.

In our simulation with the elastic contact force given by Eq. (1), $m, \sigma_{0}$ and $k$ are set to be unity, and all quantities are converted to dimensionless forms, where the unit of time scale is $\sqrt{m \sigma_{0}^{1-\Delta} / k}$. In the case of the contact force given by Eqs. (16) and (17), $\epsilon$ in Eq. (17) is set to be unity instead of $k$, and the unit of time scale is $\sqrt{m \sigma_{0}^{2} / \epsilon}$. We adopt the elastic constant $k=1.0$ or $\epsilon=1.0$, and the viscous constant $\eta=1.0$ for most of cases except for nearly elastic cases in Sec. IIE This situation corresponds to the constant restitution coefficient $e=0.043$ for the linear spring model. We use the leap-frog algorithm, which is second-order accurate in time, by using the time interval $\Delta t=0.2$ for the cases of the contact force given by Eq. (1) with checking the convergence until $\Delta t=0.05$. In the simulation with the contact force given by Eqs. (16) and (17), we use $\Delta t=0.01$.

\section{B. Scaling plots}

Figures 104 show the scaling plots of the polydisperse system with $\Delta=3 / 2$ based on Eqs. (44) (77) with Eq. (15) for the various dimensions $D=2,3$, and 4 . (See Ref. 23] for the scaling plots of the polydisperse system with $\Delta=1$ ). We use Eq. (3) for the viscous contact force. Here, the number of the particles $N$ is 2000, and the shear rate $\dot{\gamma}$ is ranged between $5 \times 10^{-7}$ and $5 \times 10^{-5}$ for $D=2,3$ and between $5 \times 10^{-6}$ and $5 \times 10^{-4}$ for $D=4$. We also use the amplitudes and the adjustable parameters $\left(t_{D}, A_{t, D}, s_{D}, A_{s, D}, p_{D}, A_{p, D}, w_{D}, A_{w, D}\right)=$ $(0.01,15.0,0.03,0.02,0.02,0.3,0.1,0.12)$ for $D=2$, $(0.01,6.0,0.04,0.03,0.025,0.3,0.15,0.25)$ for $D=3$, $(0.1,0.45,0.05,0.05,0.04,0.5,0.1,0.45)$ for $D=4$. All of the data converge to the universal master curves. These results verify the validity of our theoretical predictions in Eq. (15).

In Figs. 5 and 6, we show the scaling plots of $S$ for the bidisperse system and the monodisperse system with 


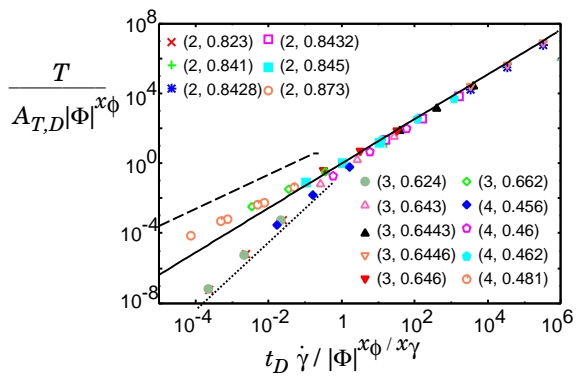

FIG. 1: (Color online) Collapsed data of the shear rate dependence of the kinetic temperature $T$ in the polydisperse system $(N=2000)$ with $\Delta=3 / 2$ using the scaling law, Eq. (4), for $D=2,3$ and 4 . The dashed line, the dotted line and the solid line are proportional to $\dot{\gamma}, \dot{\gamma}^{2}$ and $\dot{\gamma}^{x} \gamma$, respectively. The legends show the dimension $D$ and the volume fraction $\phi$ as $(D, \phi)$. The critical exponents estimated from Eq. (15) are $x_{\phi}=9 / 2$ and $x_{\gamma}=14 / 11$.

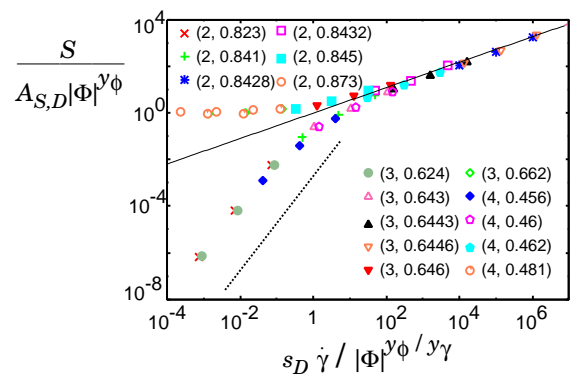

FIG. 2: (Color online) Collapsed data of the shear rate dependence of the shear stress $S$ for the polydisperse system $(N=2000)$ with $\Delta=3 / 2$ using the scaling law, Eq. (5), for $D=2,3$ and 4 . The dotted line and the solid line are proportional to $\dot{\gamma}^{2}$ and $\dot{\gamma}^{y_{\gamma}}$, respectively. The legends show the dimension $D$ and the volume fraction $\phi$ as $(D, \phi)$. The critical exponents estimated from Eq. (15) are $y_{\phi}=3 / 2$ and $y_{\gamma}=6 / 11$.

$\Delta=1$ and $D=3$, respectively, where both systems include $N=4000$ particles. The viscous contact force is given by Eq. (22), and the shear rate $\dot{\gamma}$ is ranged between $5 \times 10^{-7}$ and $5 \times 10^{-5}$. The amplitude and the adjustable parameter are $\left(s_{D}, A_{s, D}\right)=(0.035,0.04)$ for the bidisperse system and $(0.025,0.035)$ for the monodisperse system. These scaling plots support the validity of our prediction. The scaling plots for $T, P$, and $\omega$ for the bidisperse and the monodisperse systems also exhibit elegant scalings, but we omit these figures in this paper.

We have checked the validity of our scaling theory in larger systems. Figure 7 shows the scaling plot of $S$ in the three-dimensional monodisperse system with $N=20000$ particles, where the shear rate $\dot{\gamma}$ is ranged between $5 \times$ $10^{-6}$ and $5 \times 10^{-4}$. The parameters and the guide lines are the same as those for Fig. 6. This scaling supports the validity of our theory even in the larger system.

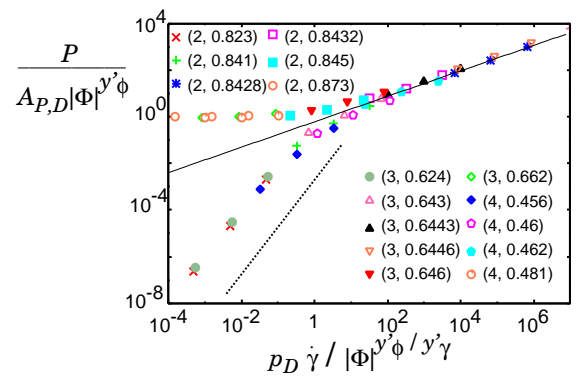

FIG. 3: (Color online) Collapsed data of the shear rate dependence of the pressure $P$ for the polydisperse system $(N=2000)$ with $\Delta=3 / 2$ using the scaling law, Eq. (6), for $D=2,3$ and 4 . The dotted line and the solid line are proportional to $\dot{\gamma}^{2}$ and $\dot{\gamma}_{\gamma}^{\prime}$, respectively. The legends show the dimension $D$ and the volume fraction $\phi$ as $(D, \phi)$. The critical exponents estimated from Eq. (15) are $y_{\phi}^{\prime}=3 / 2$ and $y_{\gamma}^{\prime}=6 / 11$.

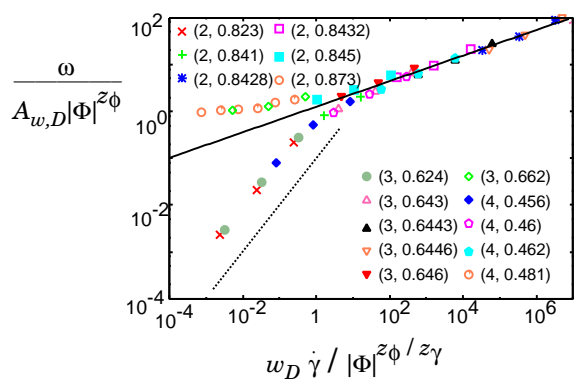

FIG. 4: (Color online) Collapsed data of the shear rate dependence of the characteristic frequency $\omega$ for the polydisperse system $(N=2000)$ with $\Delta=3 / 2$ using the scaling law, Eq. (7), for $D=2,3$ and 4 . The dotted line and the solid line are proportional to $\dot{\gamma}$ and $\dot{\gamma}^{z \gamma}$, respectively. The legends show the dimension $D$ and the volume fraction $\phi$ as $(D, \phi)$. The critical exponents estimated from Eq. (15) are $z_{\phi}=3 / 4$ and $z_{\gamma}=3 / 11$.

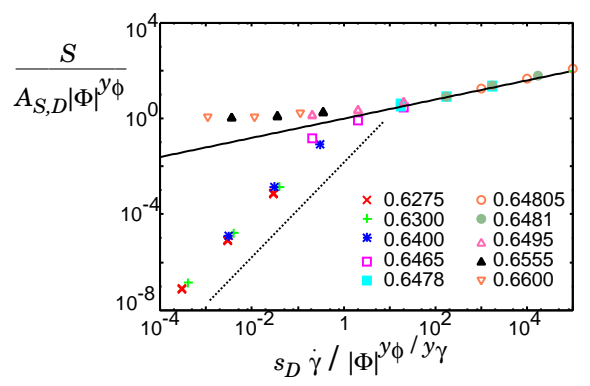

FIG. 5: (Color online) Collapsed data of the shear rate dependence of the shear stress $S$ for the bidisperse system $(N=4000)$ with $\Delta=1$ using the scaling law, Eq. (5), for $D=3$. The dotted line and the solid line are proportional to $\dot{\gamma}^{2}$ and $\dot{\gamma}^{y_{\gamma}}$, respectively. The legends represent the volume fraction $\phi$. The critical exponents estimated from Eq. (15) are $y_{\phi}=1$ and $y_{\gamma}=2 / 5$. 


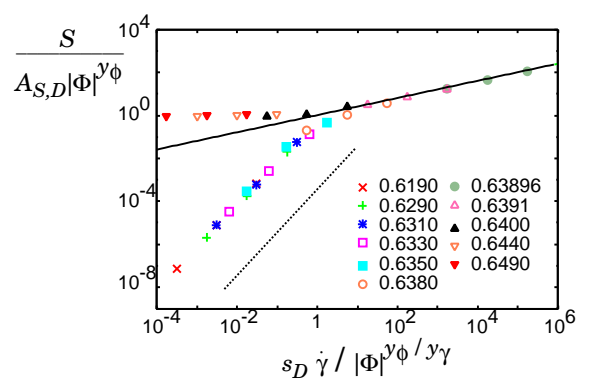

FIG. 6: (Color online) Collapsed data of the shear rate dependence of the shear stress $S$ for the monodisperse system $(N=4000)$ with $\Delta=1$ using the scaling law, Eq. (5), for $D=3$. The dotted line and the solid line are proportional to $\dot{\gamma}^{2}$ and $\dot{\gamma}^{y_{\gamma}}$, respectively. The legends show the volume fraction $\phi$. The critical exponents estimated from Eq. (15) are $y_{\phi}=1$ and $y_{\gamma}=2 / 5$.

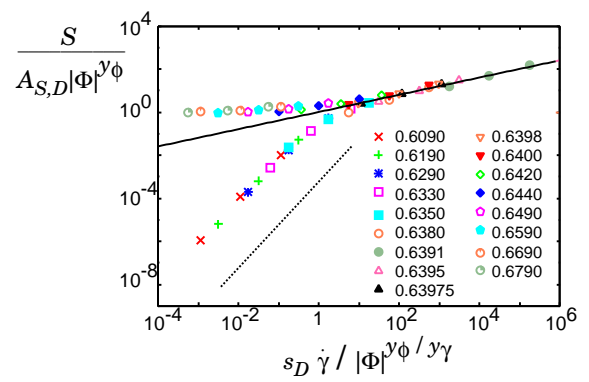

FIG. 7: (Color online) Collapsed data of the shear rate dependence of the shear stress $S$ in the larger monodisperse system ( $N=20000)$ with $\Delta=1$ using the scaling law, Eq. (5), for $D=3$. The dotted line and the solid line are proportional to $\dot{\gamma}^{2}$ and $\dot{\gamma}^{y \gamma}$, respectively. The legends show the volume fraction $\phi$. The critical exponents estimated from Eq. (15) are $y_{\phi}=1$ and $y_{\gamma}=2 / 5$.

In order to verify the validity of our theory in more general cases than that of Eq. (10), we examine the scaling plot for $S$ in the three-dimensional monodisperse system with the elastic contact force given by Eqs. (16) and (17) and the viscous contact force given by Eq. (2) in Fig. 8. Here we use the number of the particles $N=2000$, and the shear rate $\dot{\gamma}$ is ranged between $10^{-5}$ and $10^{-3}$. The amplitude and the adjustable parameter are given by $\left(s_{D}, A_{s, D}\right)=(0.004,3.0)$, where we adopt the exponents given by Eq. (15) with $\Delta=1$. The scaling in Fig. 8 supports the validity of our theory for the elastic contact force given by Eq. (16).

\section{C. $\Delta$-dependence of the critical exponents}

In order to verify the $\Delta$-dependence of the critical exponents, we plot $y_{\gamma}$ and $y_{\gamma}^{\prime}$ versus $\Delta$ for the polydisperse system with $D=2, N=4000$, and the viscous force

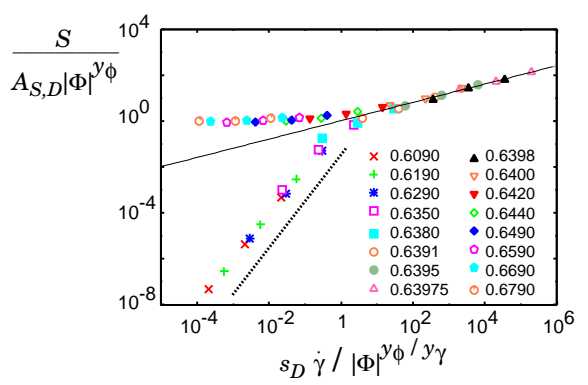

FIG. 8: (Color online) Collapsed data of the shear rate dependence of the shear stress $S$ in the monodisperse system $(N=2000)$ with the contact force given by Eqs. (16) and (17) using the scaling law, Eq. (5) for $D=3$. The dotted line and the solid line are proportional to $\dot{\gamma}^{2}$ and $\dot{\gamma}^{y_{\gamma}}$, respectively. The legends show the volume fraction $\phi$. The critical exponents estimated from Eq. (15) with $\Delta=1$ are $y_{\phi}=1$ and $y_{\gamma}=2 / 5$.

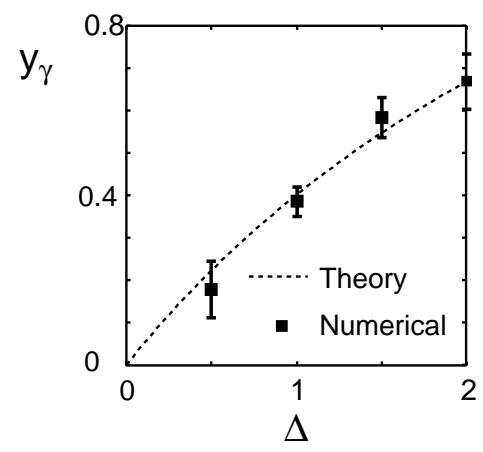

FIG. 9: Plot of $y_{\gamma}$ versus $\Delta$ for the polydisperse system $(N=$ 8000) with $D=2$.

given by Eq. (2) in Figs. 9 and 10, respectively. Here, in order to obtain Figs. 9 and 10, we have estimated $y_{\gamma}$ and $y_{\gamma}^{\prime}$, respectively, from the shear stress $S(\dot{\gamma}, \phi)$ and the pressure $P(\dot{\gamma}, \phi)$ at $\phi=\phi_{J}$ as

$$
\begin{aligned}
y_{\gamma} & =\frac{\log \left(S\left(\dot{\gamma}_{1}, \phi_{J}\right)\right)-\log \left(S\left(\dot{\gamma}_{2}, \phi_{J}\right)\right)}{\log \dot{\gamma}_{1}-\log \dot{\gamma}_{2}}, \\
y_{\gamma}^{\prime} & =\frac{\log \left(P\left(\dot{\gamma}_{1}, \phi_{J}\right)\right)-\log \left(P\left(\dot{\gamma}_{2}, \phi_{J}\right)\right)}{\log \dot{\gamma}_{1}-\log \dot{\gamma}_{2}},
\end{aligned}
$$

for $\left(\dot{\gamma}_{1}, \dot{\gamma}_{2}\right)=\left(5 \times 10^{-7}, 1.5 \times 10^{-6}\right),\left(1.5 \times 10^{-6}, 5 \times\right.$ $\left.10^{-6}\right),\left(5 \times 10^{-6}, 1.5 \times 10^{-5}\right),\left(1.5 \times 10^{-5}, 5 \times 10^{-5}\right),(5 \times$ $\left.10^{-7}, 5 \times 10^{-6}\right),\left(5 \times 10^{-6}, 5 \times 10^{-5}\right)$, and plotted the averages of $y_{\gamma}$ and $y_{\gamma}^{\prime}$ for different $\left(\dot{\gamma}_{1}, \dot{\gamma}_{2}\right)$ with the error bars, whose lengths are twice of the standard deviation of $y_{\gamma}$ and $y_{\gamma}^{\prime}$. The exponent $y_{\gamma}$ reasonably agrees with the prediction Eq. (15) in the wide range of $\Delta$. Although $y_{\gamma}^{\prime}$ is a little deviated from the theoretical prediction, we believe that the deviation becomes smaller if we use the data for smaller $\dot{\gamma}$ and larger $N$.

Figures 11 and 12 demonstrate whether the exponents for $S$ predicted by Eq. (15) can be used for the scaling 


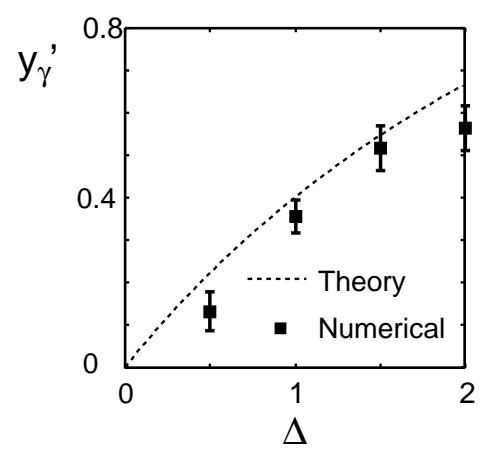

FIG. 10: Plot of $y_{\gamma}^{\prime}$ versus $\Delta$ for the polydisperse system $(N=8000)$ with $D=2$.

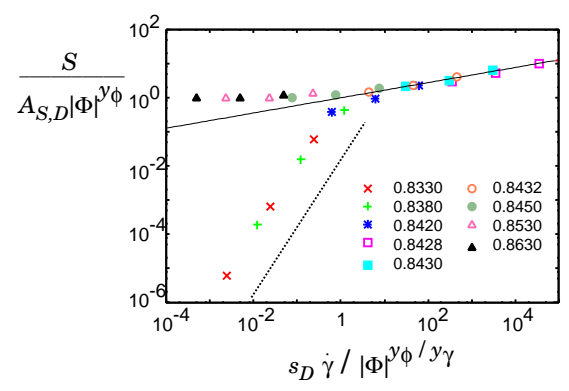

FIG. 11: (Color online) Collapsed data of the shear rate dependence of the shear stress $S$ for the polydisperse system $(N=8000)$ with $\Delta=0.5$ using the scaling law, Eq. (5) , for $D=2$. The dotted line and the solid line are proportional to $\dot{\gamma}^{2}$ and $\dot{\gamma}^{y \gamma}$, respectively. The legends show the volume fraction $\phi$. The critical exponents are estimated from Eq. (15) as $y_{\phi}=1 / 2$ and $y_{\gamma}=2 / 9$.

plots of $\Delta=0.5$ and 2 , respectively. The shear rate $\dot{\gamma}$ is ranged between $5 \times 10^{-7}$ and $5 \times 10^{-5}$. The amplitude and the adjustable parameter are $\left(s_{D}, A_{s, D}\right)=(0.015,0.05)$ for $\Delta=0.5$ and $(0.05,0.02)$ for $\Delta=2.0$, respectively. These scalings in Figs. 11 and 12 as well as the evaluated exponents by Eqs. (20) and (21) strongly support the validity of the theoretical predictions in Eq. (15) for arbitrary $\Delta$. Thus, our theory can be used for any $\Delta$.

\section{D. $\Phi$-dependence of critical variables}

Here, we examine $\Phi$-dependences of quantities predicted in Eqs. (12) and (13) with Eq. (15). First, we plot $\omega$ versus $|\Phi|$ in the jammed phase for the two-dimensional polydisperse systems $(N=2000)$ with $\Delta=3 / 2$ in Fig. [13. We adopt Eq. (2) for the viscous contact force. Note that the corresponding results for $\Delta=1$ have been reported in [23]. As the shear rate $\dot{\gamma}$ decreases, $\omega$ approaches $\omega \sim|\Phi|^{3 / 4}$ as predicted in Eq. (13) with Eq. (15). There is a plateau in the small $\dot{\gamma}$ region, but the value of it decreases as $\dot{\gamma}^{z_{\gamma}}$ in the limit $\dot{\gamma} \rightarrow 0$, which can

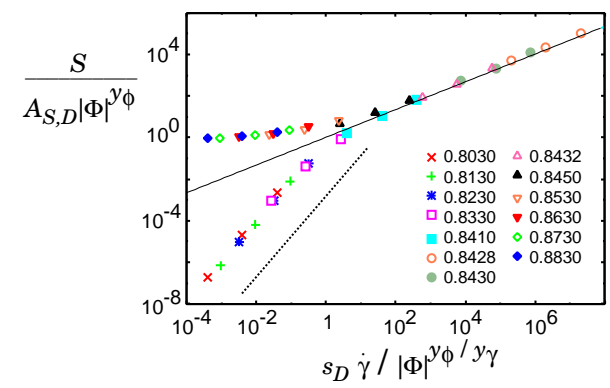

FIG. 12: (Color online) Collapsed data of the shear rate dependence of the shear stress $S$ for the polydisperse system $(N=8000)$ with $\Delta=2.0$ using the scaling law, Eq. (5), for $D=2$. The dotted line and the solid line are proportional to $\dot{\gamma}^{2}$ and $\dot{\gamma}^{y_{\gamma}}$, respectively. The legends show the volume fraction $\phi$. The critical exponents are estimated from Eq. (15) as $y_{\phi}=2$ and $y_{\gamma}=2 / 3$.

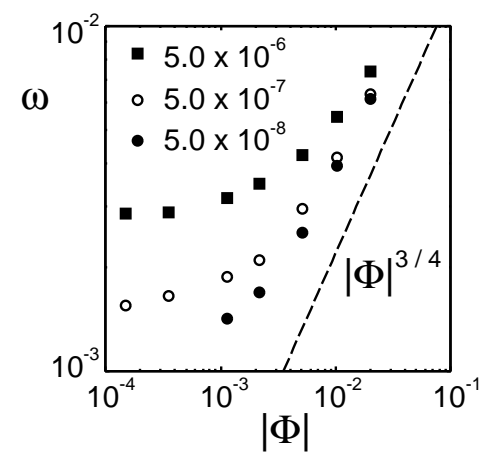

FIG. 13: Plots of $\omega$ versus $\Phi$ of the polydisperse systems for $D=2$ and $\Delta=3 / 2$ with $\dot{\gamma}=5 \times 10^{-6}, 5 \times 10^{-7}$, and $5 \times 10^{-8}$.

be predicted from Eq. (17) because $\mathcal{W}_{+}(x) \sim x^{z_{\gamma}}$ with $x=\dot{\gamma} /|\Phi|^{z_{\phi} / z_{\gamma}} \rightarrow \infty$.

Next, we show $\Phi$-dependence of the viscosity $\mu \equiv S / \dot{\gamma}$ in the unjammed phase, which is predicted as $\mu \sim$ $\dot{\gamma}|\Phi|^{-4} \sim|\Phi|^{-4}$ from Eqs. (12) and (15). We note that the critical exponent for $\mu$ is independent of $\Delta$ and $D$. Figure 14 includes the data of $\mu / \dot{\gamma}$ as a function of $|\Phi|$ for the polydisperse system $(N=2000)$ with $\Delta=1$ and $3 / 2$. We respectively adopt Eqs. (2) and (3) for the viscous contact forces in in the cases of $\Delta=1$ and $3 / 2$. Both of the data for $\Delta=1$ and $3 / 2$ satisfy the theoretical prediction $\mu / \dot{\gamma} \sim|\Phi|^{-4}$ as predicted, although there is a plateau when $|\Phi| \rightarrow 0$. The value of the plateau proportional to $\dot{\gamma}^{y_{\gamma}-2}$ can be understood by Eq. (5) [23].

There are some previous studies on the divergence of the viscosity $\mu$. For example, Losert et al. [27] observed exponents larger than 1 from their experiment. The critical exponent of the shear viscosity for foams and emulsions in Ref. [6] is between 1 and 2. For colloidal suspensions, the viscosity is believed to diverge as $|\Phi|^{-2}$ [29]. Garcia-Rojo et al. [28] reported that the scaled viscosity diverges as $\mu / \sqrt{T} \sim\left(\phi_{\mu}-\phi\right)^{-1}$ for 


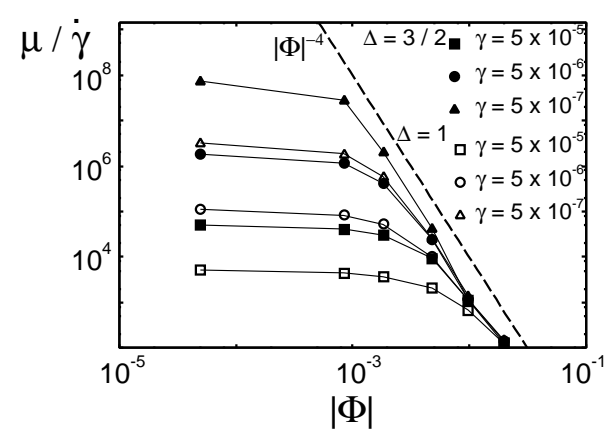

FIG. 14: Plots of $\mu / \dot{\gamma}$ versus $\Phi$ of the two-dimensional polydisperse systems for and $\Delta=1$ and $3 / 2$ with $\dot{\gamma}=$ $5 \times 10^{-5}, 5 \times 10^{-6}$, and $5 \times 10^{-7}$.

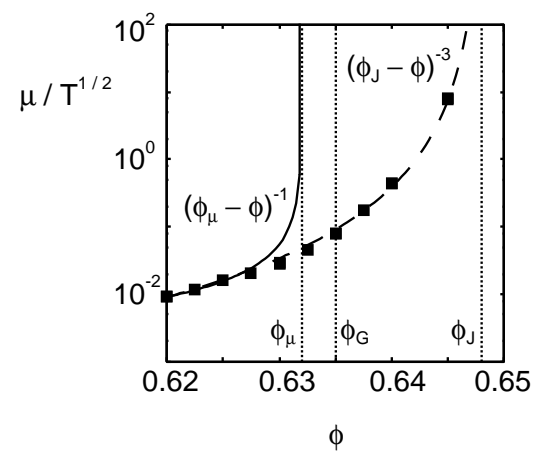

FIG. 15: $\mu / \sqrt{T}$ as a function of $\phi$ for the three-dimensional bidisperse system $(N=4000)$ with $\Delta=1$ and $\dot{\gamma}=5 \times 10^{-7}$, where the solid line and the dashed line are proportional to $\left(\phi_{\mu}-\phi\right)^{-1}$ and $\left(\phi_{J}-\phi\right)^{-3}$, respectively.

$\phi_{\mu}<\phi_{J}$ in two-dimensional elastic monodispersed harddisks. This result is contrast to our prediction that the viscosity scaled by the temperature $\sqrt{T}$ diverges at point $\mathrm{J}$ as $\mu / \sqrt{T} \sim\left(\phi_{J}-\phi\right)^{-3}$, obtained from Eq. (12) with Eq. (15).

In order to clarify whether our prediction is valid for sheared frictionless granular materials in the vicinity of the jamming transition, we examine the possibility that the viscosity satisfies $\mu / \sqrt{T} \sim\left(\phi_{\mu}-\phi\right)^{-1}$ with a fitting parameter $\phi_{\mu}$ [28] in Fig. 15 for the bidisperse system $(N=4000)$ with $D=3, \Delta=1$ and $\dot{\gamma}=5 \times 10^{-7}$. Actually we can fit the data of our three-dimensional simulation by $\mu / \sqrt{T} \sim\left(\phi_{\mu}-\phi\right)^{-1}$, but the viscosity is still finite even for $\phi>\phi_{\mu}=0.632$. We, thus, conclude that the viscosity $\mu$ in the sheared granular materials does not satisfy $\mu / \sqrt{T} \sim\left(\phi_{\mu}-\phi\right)^{-1}$, and the behavior of $\mu$ is consistent with our prediction. Here, we should note that our theory is no longer valid for the two-dimensional monodisperse system, where the shear band occurs (Fig. 16), which is not assumed in our theory.

Berthier and Witten [25, 30] reported that the relaxation time in the zero-temperature limit of the three-

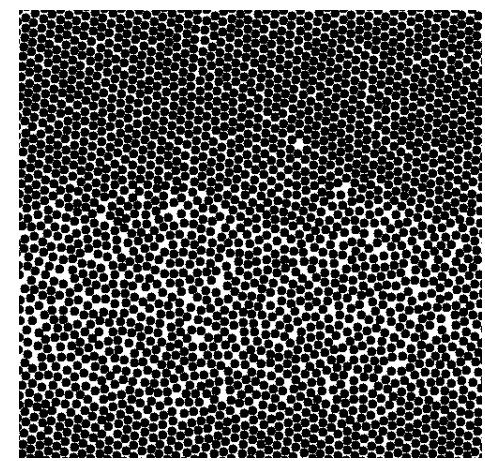

FIG. 16: The snapshot of the two-dimensional monodisperse system $(N=2401)$ with $\Delta=1, k=1.0$, and $\eta=0.00225$. We use Eq. (2) for the viscous contact force. The shear rate is $\dot{\gamma}=5 \times 10^{-5}$, and the density is $\phi=0.80$.

dimensional equilibrium bidisperse system diverges at $\phi_{G}=0.635$, which is smaller than $\phi_{J}=0.639$. Although this idea might be attractive to characterize universal feature of the jamming transition, we could not find such divergence, as shown in Fig. 15, for frictionless sheared granular materials near the jamming transition.

\section{E. Nearly elastic cases}

One might think that our scaling theory is only valid when the dissipation of the system is strong enough. Indeed, we have used the viscous constant $\eta=1.0$, which corresponds to the restitution coefficient $e=0.043$ for the linear spring model. In order to check the validity of our theory in the nearly elastic system $(e \simeq 1$ ), we perform the numerical simulation of the three-dimensional monodisperse system with $\Delta=1$ and $\eta=0.018$, which corresponds to the restitution coefficient $e=0.96$. We use the viscous contact force in Eq. (2) with the number of particles $N=2000$. The shear rate $\dot{\gamma}$ is ranged between $5 \times 10^{-6}$ and $5 \times 10^{-4}$. The amplitude and the adjustable parameter are given by $\left(s_{D}, A_{s, D}\right)=(0.03,0.04)$. The scaling plot of $S$ in this system is shown in Fig. 17. This figure supports the validity of our scaling even in the nearly elastic system.

\section{PAIR CORRELATION FUNCTION}

In this section, we discuss the behaviors of the spatial correlations in the vicinity of the jamming transition. In particular, we focus on the critical behaviors at the first peak of the pair correlation function. We restrict our interest to the monodisperse system where each particle has an identical diameter $\sigma_{0}$.

Let us discuss the spatial correlation of the density field. Figures 18 and 19 present the isotropic parts of the structure factor $S(k)$ and the pair correlation func- 


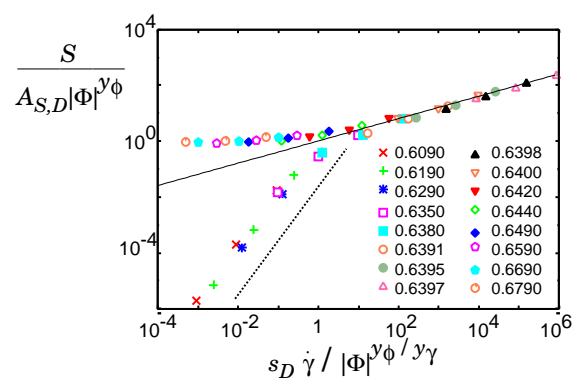

FIG. 17: (Color online) Collapsed data of the shear rate dependence of the shear stress $S$ in the nearly elastic monodisperse system $(N=2000)$ with $\Delta=1$ using the scaling law, Eq. (5), for $D=3$. The the dotted line and the solid line are proportional to $\dot{\gamma}^{2}$ and $\dot{\gamma}^{y_{\gamma}}$, respectively. The legends show the volume fraction $\phi$. The critical exponents estimated from Eq. (15) are $y_{\phi}=1$ and $y_{\gamma}=2 / 5$.

tion $g(r)$ for the three-dimensional monodisperse system, respectively. Here, $g(r)$ and $S(k)$ respectively satisfy [31]

$$
\begin{aligned}
g(r) & =\frac{1}{S_{D} r^{D-1} n}\left\langle\frac{1}{N} \sum_{i} \sum_{j \neq i} \delta\left(r-r_{i j}\right)\right\rangle, \\
S(k) & =1+(2 \pi)^{D / 2} n \int_{0}^{\infty} d r r^{D-1} g(r) \frac{J_{D / 2-1}(k r)}{(k r)^{D / 2-1}}(23)
\end{aligned}
$$

where $J_{D / 2-1}(k r)$ is the Bessel function, $S_{D}$ is the surface area of the $D$-dimensional unit sphere given by $S_{D}=$ $2 \pi^{D / 2} / \Gamma(D / 2)$ with the Gamma function $\Gamma(D / 2)$. We use $\Delta=1$, the viscous contact force given by Eq. (2), and the number of the particles $N=20000$. Because the isotropic parts of the spatial correlations are dominant in our system, we only focus on the critical properties of the isotropic parts in this section. The first peak of $g(r)$ is larger than 3 which is the maximum value of the vertical axis of Fig. 19, From Figs. 18 and 19, it seems that there is no obvious dependence of $S(k)$ and $g(r)$ on $\dot{\gamma}$ and $\phi$, but the height of the first peak $g_{0}$ of $g(r)$ strongly depends on $\dot{\gamma}$ and $\phi$. We plot the dependence of the first peak of $g(r)$ on $\dot{\gamma}$ for small $r-\sigma_{0}$ region in Fig. 20, in which the first peak $g_{0}$ becomes higher and the width of the half-height of the first peak $h_{0}$ becomes narrower as $\dot{\gamma}$ decreases.

The dependence of the peak on $\phi$ and $\dot{\gamma}$ can be roughly estimated from our scaling law for the pressure $P$ in Eq. (6). Indeed, the coordination number $Z$ and the pressure $P$, respectively, satisfy

$$
\begin{aligned}
& Z=\frac{S_{D} n}{2} \int_{0}^{\sigma_{0}} d r r^{D-1} g(r), \\
& P \simeq \frac{S_{D} n^{2} k}{2} \int_{0}^{\sigma_{0}} d r r^{D}\left(\sigma_{0}-r\right)^{\Delta} g(r) .
\end{aligned}
$$

The derivation of these equations is briefly explained in Appendix B. Since the first peak near $\sigma_{0}$ is characterized

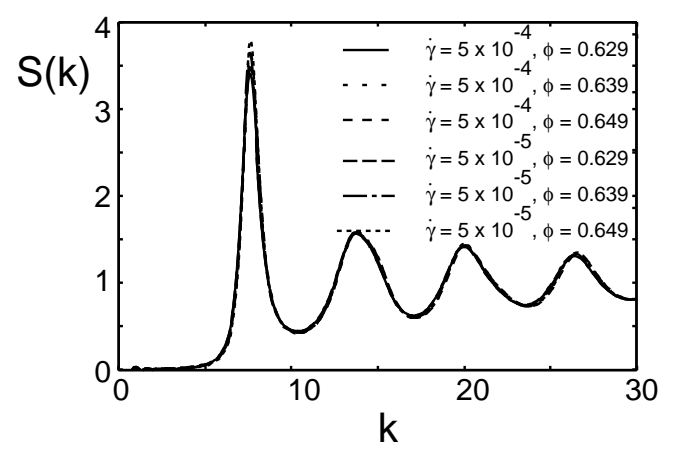

FIG. 18: Structure factor $S(k)$ in the threedimensional monodisperse system $(N=20000)$ with $\phi=0.629,0.639,0.649$ and $\dot{\gamma}=5 \times 10^{-4}$ and $5 \times 10^{-5}$.

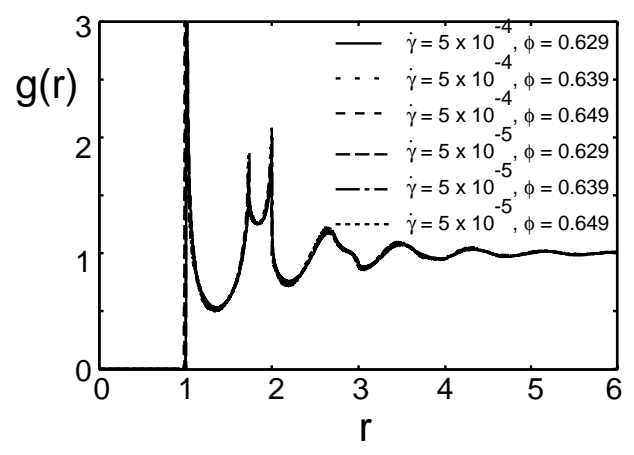

FIG. 19: Pair correlation function $g(r)$ in the threedimensional monodisperse system $(N=20000)$ with $\phi=$ $0.629,0.639,0.649$ and $\dot{\gamma}=5 \times 10^{-4}$ and $5 \times 10^{-5}$.

by the peak value $g_{0}$ and the width $h_{0}$, Eqs. (24) and (25) are approximated by

$$
\begin{aligned}
Z & \sim S_{D} n \int_{\sigma_{0}-h_{0}}^{\sigma_{0}} r^{D-1} g_{0} \\
& \sim g_{0} h_{0}\left\{1+O\left(h_{0}\right)\right\}, \\
P & \sim S_{D} n^{2} \int_{\sigma_{0}-h_{0}}^{\sigma_{0}} d r r^{D} k\left(\sigma_{0}-r\right)^{\Delta} g_{0}, \\
& \sim h_{0}^{\Delta+1} g_{0}\left\{1+O\left(h_{0}\right)\right\} .
\end{aligned}
$$

With the aid of Eq. (26), we find that $g_{0}$ and $h_{0}$ satisfy the relation near the point $\mathrm{J}$

$$
g_{0} h_{0} \sim \text { const. }
$$

where we have used the known result on the coordination number $Z \simeq 2 D$ for the frictionless granular particles near the point J. Substituting Eq. (28) into Eq. (27), we obtain the relation between $g_{0}$ and the pressure $P$

$$
g_{0} \sim P^{-1 / \Delta} .
$$

From Eqs. (12), (15) and (29), the height of the first peak value $g_{0}$ in the unjammed phase is given by

$$
g_{0} \sim \dot{\gamma}^{-2 / \Delta}|\Phi|^{4 / \Delta} \text {. }
$$




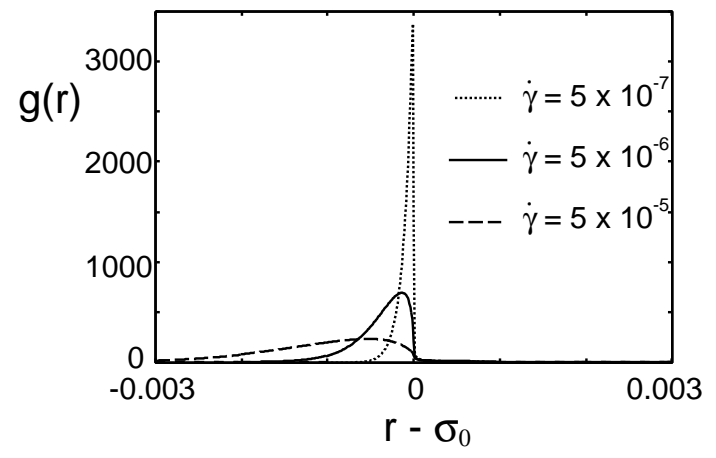

FIG. 20: The first peak of pair correlation function $g(r)$ in the three-dimensional monodisperse system $(N=20000)$ with $\Delta=1$ and $\phi=0.639$ for various shear rates $\dot{\gamma}$.

Similarly, from Eqs. (10), (15) and (29) $g_{0}$ satisfies

$$
g_{0} \sim|\Phi|^{-1}
$$

in the jammed phase, which is consistent with the numerical result of the unsheared jammed system [19]. At the critical point, i.e. $\Phi=0$, thus, $g_{0}$ is given by

$$
g_{0} \sim \dot{\gamma}^{-2 /(\Delta+4)}
$$

from Eqs. (11), (15), and (29).

In order to check our predictions in Eqs. (30)-(32), we plot $g_{0}$ versus $\dot{\gamma}$ for various densities in Fig. 21 The numerical data are consistent with the theoretical prediction in which $g_{0}$ is proportional to $\dot{\gamma}^{-2 / \Delta}$ in the unjammed phase (see Eq.(30)), but $g_{0}$ is almost a constant in the jammed phase (see Eq.(31)), and $g_{0}$ satisfies $g_{0} \sim \dot{\gamma}^{-2 /(\Delta+4)}$ at point J (see Eq.(32) $)$.

Figure 22 examines the validity of Eq. (31) in the zero shear limit of the jammed phase, in which $g_{0}$ seems to satisfy $g_{0} \sim 1 /|\Phi|$. On the other hand, $g_{0}$ tends to satisfy $g_{0} \dot{\gamma}^{2 / \Delta} \sim|\Phi|^{\Delta / 4}$ in the unjammed phase as in Eq. (30) (see Fig. 23).

\section{DISCUSSION AND CONCLUSION}

This section consists of two parts. In the first part, we will discuss our results, and we will conclude our work in the second part. In the first part, let us compare our results with those by Hatano [21], discuss the growing length scale near point $J$, the long-range spatial correlation, and the scaling laws for the Langevin dynamics and the frictional particles.

\section{A. Discussion}

Let us compare our results with those by Hatano 21]. Hatano estimated the values of the exponents as

$$
\begin{aligned}
x_{\Phi} & =2.5, \quad x_{\gamma}=1.3, \quad y_{\Phi}=1.2, \\
y_{\gamma} & =0.63, \quad y_{\Phi}^{\prime}=1.2, \quad y_{\gamma}^{\prime}=0.57,
\end{aligned}
$$

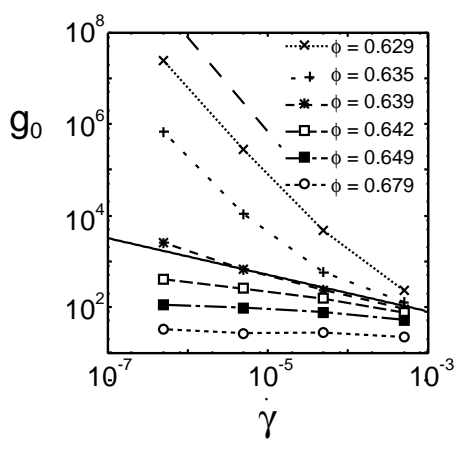

FIG. 21: The height of the first peak $g_{0}$ of $g(r)$ as a function of $\dot{\gamma}$ for the three-dimensional monodisperse systems $(N=$ $20000)$ with $\Delta=1$ for various densities $\phi$. The critical density is $\phi_{J}=0.639$. The solid line is proportional to $\dot{\gamma}^{-2 /(\Delta+4)}$. The dashed line is proportional to $\dot{\gamma}^{-2 / \Delta}$.

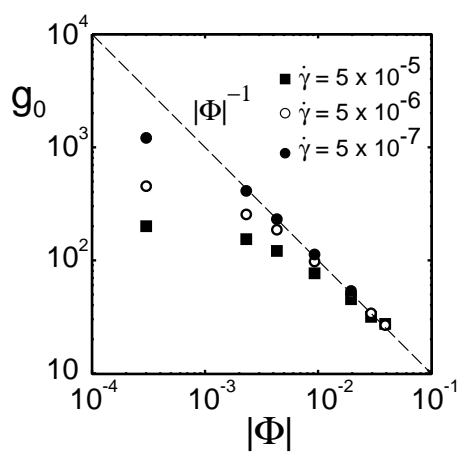

FIG. 22: The plots of $g_{0}$ versus $|\Phi|$ in the jammed phase for the three-dimensional monodisperse system $(N=20000)$ with $\Delta=1$ for various shear rates $\dot{\gamma}$. The dashed guide line is proportional to $|\Phi|^{-1}$.

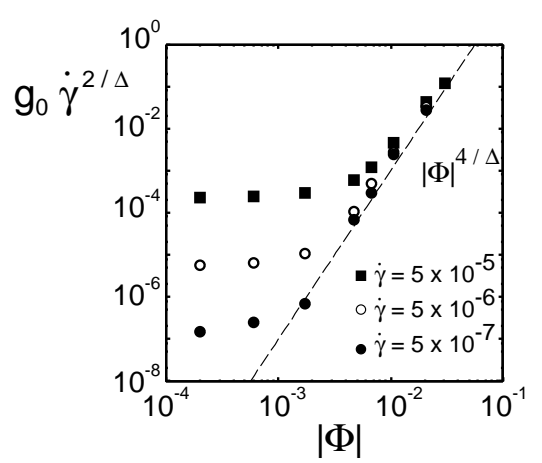

FIG. 23: The plots of $g_{0} \dot{\gamma}^{2 / \Delta}$ versus $|\Phi|$ in the unjammed phase for the monodisperse system $(N=20000)$ with $\Delta=1$ for various shear rates $\dot{\gamma}$. The dashed guide line is proportional to $|\Phi|^{4 / \Delta}$. 
for $\Delta=1$, and

$$
\begin{aligned}
x_{\Phi} & =3.2, \quad x_{\gamma}=1.3, \quad y_{\Phi}=1.8, \\
y_{\gamma} & =0.75, \quad y_{\Phi}^{\prime}=1.8, \quad y_{\gamma}^{\prime}=0.72,
\end{aligned}
$$

for $\Delta=3 / 2$. The system analyzed in Ref. [21] corresponds to our polydisperse system, but these values are different from those in Eq. (15). Here, let us clarify the origin of the differences. (i) The estimation of the critical exponents strongly depends on the range of $\dot{\gamma}$. The value of $\phi_{J}$ and the range of $\dot{\gamma}$ in Ref. 21] are larger than ours. If we adopt $\phi_{J}$ and the range of $\dot{\gamma}$ in Ref. 21], we can recover his scaling laws in Eqs. (33) and (34) as shown in Fig. 24, while we find the obvious violation of his scaling (Fig. 25) in the small $\dot{\gamma}$ region $\left(\dot{\gamma}<10^{-4}\right)$. On the other hand, our scaling is still valid as shown in Fig. 26 for $\dot{\gamma}<10^{-4}$. In order to extract the critical properties, it is needless to say that we should use the data in the small $\dot{\gamma}$ region. Hence, our prediction for the exponents is more appropriate than Hatano's estimation. (ii) The estimated exponents in Eqs. (33) and (34) cannot be valid even when we adjust the value of $\phi_{J}$ as the fitting parameter in the small $\dot{\gamma}$ region. The value of $\phi_{J}$ is well established by the simulation of the sphere packing for the bidisperse system and the monodisperse system [18, 19]. The scaling plots using the estimated value of $\phi_{J}$ (Figs. 5 and 6) evidently support the validity of our prediction.

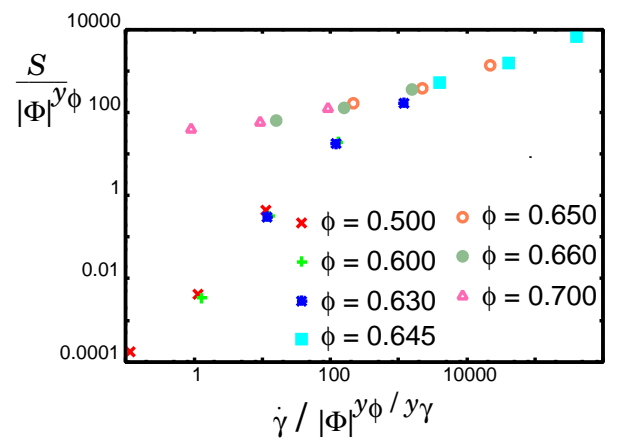

FIG. 24: The scaling of the shear stress $S /|\Phi|^{y_{\phi}}$ for the polydisperse system $(N=4000)$ with $\Delta=1$ using Eq. (33) as a function of the scaled shear rate $\dot{\gamma} /|\Phi|^{y_{\phi} / y_{\gamma}}$ for $10^{-4} \leq$ $\dot{\gamma} \leq 10^{-1}, 0.5 \leq \phi \leq 0.7$ with $D=3$.

There are some studies to indicate the diverging time scale near point $J[13,20]$. Indeed, in the scaling relations of $T, S, P$, and $\omega$ in Eqs. (4)-(7) with Eq. (15), the shear rate $\dot{\gamma}$ is scaled by the same diverging time scale $\tau \sim|\Phi|^{-(\Delta+4) / 2}$. For conventional critical phenomena, the divergence of the time scale is associated with the diverging length scale. Therefore, there are many papers to discuss growing length scale in the vicinity of the jamming transition [6, 8, 9 , 10, 11, 12, 13, 20, 32]. However, the length scale in the previous studies is as much as the size of several particles in their papers, and it is not clear whether the length scale diverges at point J. We, thus,

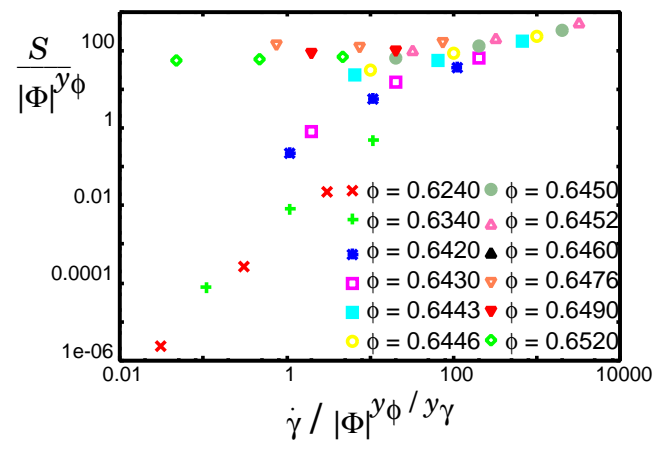

FIG. 25: The scaling of the shear stress $S /|\Phi|^{y_{\phi}}$ for the polydisperse system $(N=4000)$ with $\Delta=1$ using Eq. (33) as a function of the scaled shear rate $\dot{\gamma} /|\Phi|^{y_{\phi} / y_{\gamma}}$ for $5 \times 10^{-7} \leq$ $\dot{\gamma} \leq 5 \times 10^{-5}, 0.624 \leq \phi \leq 0.652$ with $D=3$.

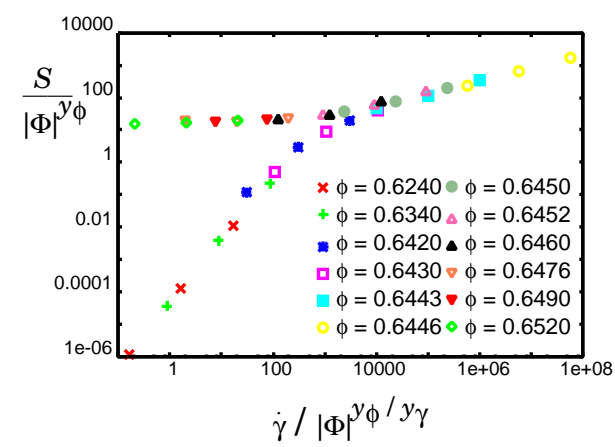

FIG. 26: The scaling of the shear stress $S /|\Phi|^{y_{\phi}}$ for the polydisperse system $(N=4000)$ with $\Delta=1$ using Eq. (15) as a function of the scaled shear rate $\dot{\gamma} /|\Phi|^{y_{\phi} / y_{\gamma}}$ for $5 \times 10^{-7} \leq$ $\dot{\gamma} \leq 5 \times 10^{-5}, 0.624 \leq \phi \leq 0.652$ with $D=3$.

conjecture that the length scale might be unrelated to determine the critical exponents. The success of our mean field theory supports the validity of this conjecture.

In relatively dilute sheared systems, we know the existence of the long-range correlation [33, 34, 35, 36]. The existence of the long-range correlation is only confirmed in the relatively dilute systems such as $\phi \leq 0.50$ for the three dimensional case. We, thus, still do not know whether there is the long-range correlation in the jammed systems, and the role of the correlation. We will discuss the long-range correlation in the jammed systems elsewhere.

The similar scaling relations with different values of the exponents are observed in the zero temperature limit of Langevin thermostat system, where the Newtonian law $S \propto \dot{\gamma}$ is held in the unjammed region [6]. However, we cannot extend our simple theory to this system because the characteristic frequency defined by $\omega=\dot{\gamma} S /(n T)$ is always constant in this system, which differs from the scaling relation (77). We will discuss the results of this situation elsewhere. 
In this paper, we restrict our interest to the frictionless particles. When the particles have friction, the situation will be changed completely. For examples, the critical density $\phi_{J}$ of the frictional particles in the static granular packings, becomes smaller than that of the frictionless particles [37], and depends on the packing process [26, 38]. Thus, critical properties of the scaling in the sheared dynamical systems of frictional particles should differ from the frictionless assemblies. This also will be our future work.

\section{B. Conclusion}

In conclusion, we have extensively checked the validity of the mean field theory proposed in Ref. [23] numerically, and we demonstrate that most of all our numerical results are consistent with the theoretical predictions in Eq. (15). Thus, we may conclude that the jamming transition for frictionless sheared granular materials is a continuous transition in which the critical exponents are independent of the spatial dimension and are determined by the local elastic force between contacted grains. We also confirm that the viscosity diverges at point $\mathrm{J}$ as $\left(\phi_{J}-\phi\right)^{-4}$. Essential new findings beyond Ref. [23] are the critical behaviors of the first peak of the pair correlation function given by Eqs. (30), (31) and (32), which are also consistent with our numerical simulation.

\section{Acknowledgments}

We thank S. Luding, S. Sasa, B. Kim, and S.-H. Chong for the valuable discussion. This work is partially supported by Ministry of Education, Culture, Science and Technology (MEXT), Japan (Grant Nos. 21015016, 21540384 and 21540388) and the Grant-in-Aid for the global COE program "The Next Generation of Physics, Spun from Universality and Emergence" from the Ministry of Education, Culture, Sports, Science and Technology (MEXT) of Japan. The numerical calculations were carried out on Altix3700 BX2 at YITP in Kyoto University.

\section{APPENDIX A: DERIVATION OF THE VALUES OF THE CRITICAL EXPONENTS}

In this appendix, we theoretically determine the values of the critical exponents in Eq (15). The derivation is parallel to that in Ref. [23], but contains some generalizations with the help of a simplified argument.

At first, we should note that the scaling functions $\mathcal{T}_{ \pm}(x), \mathcal{S}_{ \pm}(x), \mathcal{P}_{ \pm}(x)$, and $\mathcal{W}_{ \pm}(x)$ satisfy

$$
\begin{array}{ll}
\lim _{x \rightarrow 0} \mathcal{T}_{+}(x)=x, & \lim _{x \rightarrow 0} \mathcal{S}_{+}(x)=1, \\
\lim _{x \rightarrow 0} \mathcal{P}_{+}(x)=1, & \lim _{x \rightarrow 0} \mathcal{W}_{+}(x)=1,
\end{array}
$$

$$
\begin{aligned}
& \lim _{x \rightarrow 0} \mathcal{T}_{-}(x)=x^{2}, \quad \lim _{x \rightarrow 0} \mathcal{S}_{-}(x)=x^{2}, \\
& \lim _{x \rightarrow 0} \mathcal{P}_{-}(x)=x^{2}, \quad \lim _{x \rightarrow 0} \mathcal{W}_{-}(x)=x, \\
& \lim _{x \rightarrow \infty} \mathcal{T}_{ \pm}(x)=x^{x_{\gamma}}, \quad \lim _{x \rightarrow \infty} \mathcal{S}_{ \pm}(x)=x^{y_{\gamma}} \\
& \lim _{x \rightarrow \infty} \mathcal{P}_{ \pm}(x)=x^{y_{\gamma}^{\prime}}, \lim _{x \rightarrow \infty} \mathcal{W}_{ \pm}(x)=x^{z_{\gamma}}
\end{aligned}
$$

The scaling relations (12)-(14) are obtained from Eqs. (A1) - (A3) with Eqs. (4)-(7).

Let us assume that the inverse of the shear rate $\dot{\gamma}^{-1}$ can be scaled by a characteristic time scale. Therefore we can assume that the ratios between the exponents $x_{\phi} / x_{\gamma}$, $y_{\phi} / y_{\gamma}, y_{\phi}^{\prime} / y_{\gamma}^{\prime}$ and $z_{\phi} / z_{\gamma}$ in the scaling laws Eqs. (4)-(7) are common as

$$
\alpha=\frac{x_{\phi}}{x_{\gamma}}=\frac{y_{\phi}}{y_{\gamma}}=\frac{y_{\phi}^{\prime}}{y_{\gamma}^{\prime}}=\frac{z_{\phi}}{z_{\gamma}} .
$$

In other words, the characteristic time scale $\tau$ exhibits critical slowing down as $\tau \sim|\Phi|^{-\alpha}$. This property has already been indicated by Ref. 21]. It should be noted that we can determine the exponents without this assumption 23].

Substituting Eq. (12) into Eq. (11) with Eq. (A4), we obtain the relation between the exponents as

$$
z_{\phi}=y_{\phi}-x_{\phi}+\alpha .
$$

Let us assume that the pressure in the jammed phase $(\Phi>0$ and $\dot{\gamma} \rightarrow 0)$ converges to that of unsheared jammed phase satisfying $P \sim \Phi^{\Delta}$ [19]. Hence, comparing the scaling property of $P$ in Eq. (13) with $P \sim \Phi^{\Delta}$, we find the relation

$$
y_{\phi}^{\prime}=\Delta \text {. }
$$

We also assume that Coulomb's frictional law is held in granular systems [39]. Thus, $S / P$ is independent of $\Phi$ [23] and we obtain

$$
y_{\phi}=y_{\phi}^{\prime}
$$

with the aid of Eq. (13).

We can use the properties of the cut off frequency $f_{c}$ in the density of state in the jammed phase, which satisfies $f_{c} \sim \sqrt{P}[20]$. Since we expect that there is only one time scale, it is reasonable to assume that the characteristic frequency $\omega$ in the limit $\dot{\gamma} \rightarrow 0$ can be scaled by the cutoff frequency $f_{c}$. Thus, we obtain

$$
z_{\phi}=\frac{1}{2} y_{\phi}^{\prime} .
$$

Finally, let us use a similar argument on the characteristic frequency $\omega$ in the unjammed phase to that in the jammed phase. Since the characteristic frequency $\omega$ is estimated as $\omega \sim \sqrt{T / m} / l(\Phi)$ with the mean free path $l(\Phi)$, which is evaluated as $\left(\sigma / D \phi_{J}\right)|\Phi|$ in the vicinity of point J, we obtain

$$
x_{\phi}=2 z_{\phi}+2
$$


where we have used Eq. (12) .

From Eqs. (A5) the exponents $\alpha, x_{\phi}, y_{\phi}, y_{\phi}^{\prime}$, and $z_{\phi}$ are given by

$$
\begin{aligned}
\alpha & =\frac{\Delta+4}{2}, \quad x_{\Phi}=2+\Delta, \quad y_{\Phi}=\Delta, \\
y_{\Phi}^{\prime} & =\Delta, \quad z_{\Phi}=\frac{\Delta}{2} .
\end{aligned}
$$

The exponents $x_{\gamma}, y_{\gamma}, y_{\gamma}^{\prime}$, and $z_{\gamma}$ are obtained from Eqs. (A4) and (A10). Hence, we have determined all the critical exponents as Eq. (15).

\section{APPENDIX B: THE EXPRESSIONS OF $P$ AND $Z$ BY $g(r)$}

In this appendix, we derive Eqs. (24) and (25). The coordination number $Z$ is given by $Z=M / N$, where $M$ is the number of the points of contact. Since $M$ is given by the number of the pairs whose distances are smaller than $\sigma_{0}, Z$ is given by

$$
Z=\frac{1}{N} \int_{0}^{\sigma_{0}} d r\left\langle\frac{1}{2} \sum_{i} \sum_{j \neq i} \delta\left(r-r_{i j}\right)\right\rangle
$$

Substituting Eq. (22) into this equation, we obtain Eq. (24).

Since the contribution to the pressure $P$ from the elastic force is dominant in our system, we approximate $P$ in Eq. (10) with the aid of Eq. (22) as

$$
\begin{aligned}
P & \simeq \frac{1}{2 D V}\left\langle\sum_{i} \sum_{j \neq i} r_{i j} f_{\mathrm{el}}\left(r_{i j}\right)\right\rangle \\
& =\frac{1}{2 D V} \int_{0}^{\infty} d r r f_{\mathrm{el}}(r)\left\langle\sum_{i} \sum_{j \neq i} r_{i j} \delta\left(r-r_{i j}\right)\right\rangle \\
& =\frac{S_{D} n^{2}}{2} \int_{0}^{\infty} d r r^{D} f_{\mathrm{el}}(r) g(r)
\end{aligned}
$$

Substituting Eq. (1) into Eq. (B2), we obtain Eq. (25).
[1] H. M. Jaeger, S. R. Nagel, and R. P. Behringer, Rev. Mod. Phys. 68, 1259 (1996).

[2] P. N. Pusey, in Liquids, Freezing and the Glass Transition, Part II , Les Houches Summer School Proceedings Vol. 51, edited by J. -P. Hansen, D. Levesque, and J. Zinn-Justin (Elsevier, Amsterdam, 1991), Chap. 10.

[3] D. J. Durian and D. A. Weitz, "Foams," in Kirk-Othmer Encyclopedia of Chemical Technology, 4th ed., edited by J. I. Kroschwitz (Wiley, New York, 1994), Vol. 11, p. 783.

[4] A. J. Liu and S. R. Nagel, Nature 396, 21 (1998).

[5] T. Hatano, M. Otsuki, and S. Sasa, J. Phys. Soc. Jpn. 76, 023001 (2007).

[6] P. Olsson and S. Teitel, Phys. Rev. Lett. 99 (2007), 178001.

[7] L. Berthier and J.-L. Barrat, J. Chem. Phys. 116, 6228 (2002).

[8] A. R. Abate and D. J. Durian, Phys. Rev. E. 74, 031308 (2006).

[9] A. R. Abate and D. J. Durian, Phys. Rev. E. 76, 021306 (2007).

[10] F. Lechenault, O. Dauchot, G. Biroli, and J.-P. Bouchaud, Europhys. Lett. 83, 46003 (2008).

[11] K. Watanabe and H. Tanaka, Phys. Rev. Lett. 100, 158002 (2008).

[12] T. Hatano, e-print arXiv:0804.0477.

[13] T. Hatano, Phys. Rev. E 79, 050301 (2009).

[14] M. Fuchs and M. E. Cates, Phys. Rev. Lett. 89, 248304 (2002).

[15] K. Miyazaki, D. R. Reichman, and R. Yamamoto, Phys. Rev. E 70, 011501 (2004).

[16] S.-H. Chong, B. Kim, Phys. Rev. E 79, 021203 (2009).

[17] H. Hayakawa and M. Otsuki, Prog. Theor. Phys. 119, 381 (2008).
[18] C. S. O'Hern, S. A. Langer, A. J. Liu, and S. R. Nagel, Phys. Rev. Lett. 88, 075507 (2002).

[19] C. S. O'Hern, L. E. Silbert, A. J. Liu, and S. R. Nagel, Phys. Rev. E 68, 011306 (2003).

[20] M. Wyart, L. E. Silbert, S. R. Nagel, and T. A Witten, Phys. Rev. E 72, 051306 (2005).

[21] T. Hatano, J. Phys. Soc. Jpn. 77, 123002 (2008).

[22] H. Yoshino, T. Nogawa, and B. Kim, New J. Phys. 11, 013010 (2009) .

[23] M. Otsuki and H. Hayakawa, Prog. Theor. Phys. 121, 647 (2009).

[24] D. J. Evans and G. P. Morriss, "Statistical mechanics of nonequilibrium liquids", (Academic Press, London, 1990), Chap. 3.

[25] L. Berthier and T. A. Witten, Europhys. Lett. 86, 10001 (2009).

[26] S. Inagaki, M. Otsuki, and S. Sasa, e-print arXiv:0901.4150

[27] W. Losert, L. Bocquet, T. C. Lubensky, and J. P. Gollub, Phys. Rev. Lett. 85, 1428 (2000).

[28] R. Garcia-Rojo, S. Luding, and J. J. Brey Phys. Rev. E 74, 061305 (2006).

[29] W. B. Russel, D. A. Saville, and W. R. Schowalter, Colloidal Dispersions (Cambridge University Press, New York, 1989).

[30] L. Berthier and T. A. Witten, arXiv:0903:1934.

[31] J. P. Hansen and I. R. McDonald, Theory of Simple Liquids, (Academic Press, London, 1976).

[32] D. A. Head, Phys. Rev. Lett. 102, 138001 (2009).

[33] J. F. Lutsko and J. W. Dufty, Phys. Rev. A 32, 3040 (1985).

[34] J. F. Lutsko and J. W. Dufty, Phys. Rev. E 66, 041206 (2002). 
[35] H. Wada and S. Sasa, Phys. Rev. E 67, 065302(R) (2003).

[36] M. Otsuki and H. Hayakawa, Phys. Rev. E 79, 021502 (2009).

[37] K. Shundyak, M. van Hecke, and W. van Saarloos, Phys. Rev. E 75, 010301(R) (2007).
[38] H. P. Zhang and H. A. Makse, Phys. Rev. E 72, 011301 (2005).

[39] T. Hatano, Phys. Rev. E 75, 060301(R) (2007). 